\title{
Maxwell + TDDFT multi-scale simulation for laser-matter interactions
}

\author{
Shunsuke A. Sato ${ }^{1, *}$ and Kazuhiro Yabana ${ }^{1,2}$ \\ ${ }^{1}$ Graduate School of Pure and Applied Sciences, University of Tsukuba, Japan \\ ${ }^{2}$ Center for Computational Sciences, University of Tsukuba, Japan \\ *sato@nucl.ph.tsukuba.ac.jp
}

Received: December 28, 2013; Accepted: February 11, 2014; Published: October 31, 2014

\begin{abstract}
We are developing a theoretical and computational method to describe interactions between an intense laser pulse and a solid. The laser-solid interactions are extensively investigated in current frontiers of optical sciences. There are two spatial scales in the problem: the scale of laser wavelength typically a $\mu \mathrm{m}$ and the scale of electron dynamics in a solid less than a nm. We have developed a multi-scale simulation for this problem, describing propagation of the electromagnetic fields by the Maxwell equations and the electron dynamics in a unit cell of a solid by the time-dependent density functional theory. In calculating the coupled dynamics, we employ a finite difference scheme for both space and time variables. Two kinds of grids with different grid spacings are used for space, while a single time step is used for time. We present our theoretical framework, numerical implementation, and parallelization of our multi-scale simulation. It will be shown that high parallel efficiency and computing performance are realized in our computational code.
\end{abstract}

Keywords: First-principles calculation, Laser science, Multi-scale simulation

\section{Introduction}

In current frontiers of laser sciences, studies of interactions between a highly-intense, ultrashort laser pulse and a solid have been attracting much attention. Ultrashort laser pulses have enabled us to investigate electron dynamics in the time scale of a few femtosecond or less in solids [2,3]. Extremely intense laser pulses induce nonlinear electron dynamics in solids. The nonlinear laser-matter interactions are expected to be useful to control and manipulate electron dynamics in solids in a very short period.

In conventional descriptions, the laser-solid interactions are treated by two theories, macroscopic electromagnetism and quantum mechanics for electron dynamics. Propagation of macroscopic electromagnetic fields is described by the macroscopic Maxwell equations which include properties of a solid through susceptibilities. Linear and nonlinear susceptibilities are calculated by quantum mechanics using perturbation theories. The calculation 
of the susceptibility is one of central issues in computational materials sciences. This separation into two theories is possible by the following two facts. One is the weakness of the laser-matter interaction that allows us to use a perturbation theory in quantum mechanics. The other is a large difference in spatial scales between laser wavelength (typically $\mu \mathrm{m}$ ) and electron dynamics (typically less than a $\mathrm{nm}$ ). In the past, computational approaches have been developed independently for two theories. In macroscopic electromagnetism, Maxwell equations have been solved using for example, the finite-difference time-domain (FDTD) method. In microscopic quantum mechanics, calculations of linear and nonlinear susceptibilities such as dielectric function have been extensively achieved in the density functional and many-body perturbation theories.

The conventional description mentioned above becomes useless, however, for interactions involving extremely intense laser pulses. The strong electric field induces extremely nonlinear electron dynamics that cannot be treated by the perturbation theory of quantum mechanics. Then one cannot separately calculate macroscopic electromagnetism for electromagnetic fields and quantum mechanics for electron dynamics, but needs to solve the coupled dynamics simultaneously. Namely, we need to solve the coupled equations of Maxwell and time-dependent Schrödinger equations. Computationally, it requires to combine two kinds of simulations in a multi-scale framework: one is the FDTD calculation for macroscopic electromagnetic fields and the other is the first-principles calculation for electron dynamics based on the time-dependent density functional theory (TDDFT). This multi-scale simulation can only be possible using a very large supercomputer such as the K-computer in Kobe, Japan.

In our implementation of the multi-scale simulation, we introduce two kinds of spatial grids in Cartesian coordinates. We first introduce macroscopic grids to solve the Maxwell equations for macroscopic electromagnetic fields. At each macroscopic grid point, we introduce microscopic three-dimensional Cartesian grids to describe electron dynamics. We solve the time-dependent Kohn-Sham (TDKS) equation that is the basic equation of the TDDFT in the microscopic grids. Between macroscopic and microscopic scales, macroscopic vector potential (input for the microscopic scale) and macroscopic current (output from the microscopic scale) are exchanged. Since their amount of data are very small, this multi-scale simulation is ideally suited for calculations using massively parallel computers. We have been developing our own computer code for this simulation, named ARTED: Abinitio Real-Time Electron Dynamics simulator. As will be shown later, the ARTED achieves good computational performance up to about 100,000 cores.

The construction of the present paper is as follows. In Sec. 2, we describe theoretical framework of our multi-scale calculation. In Sec. 3, we present numerical aspects of the simulation. After explaining numerical implementation, we describe how our code is parallelized for the multi-scale calculation. We then present computational performances. In Sec. 4, a summary will be presented. 


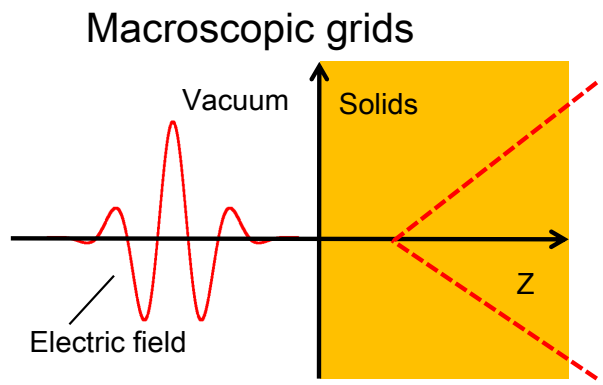

Microscopic grids

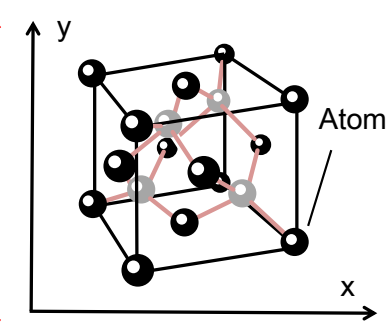

Figure 1: A schematic picture of the multi-scale coordinates system. Left-hand side shows the macroscopic coordinate to describe propagation of the macroscopic electromagnetic fields. Righ-hand side shows the microscopic coordinates to describe quantum dynamics of electrons induced by the fields.

\section{Theoretical formulation and scientific aspects}

\subsection{Theoretical framework}

To explain our multi-scale simulation, we consider a simple case: a linearly polarized laser pulse irradiating normally on a surface of bulk $\mathrm{Si}$. We take a coordinate system shown in Fig. 1 where the surface of bulk $S i$ is taken to be a $Z=0$ plane, the direction of the laser electric field is parallel to $x$-axis, and the direction of the laser propagation is parallel to $z$-axis. We denote the macroscopic coordinate in $z$-direction as $Z$. We describe the macroscopic electromagnetic field of laser pulse using a vector potential, $\vec{A}_{Z}(t)$, which is related to the electric field by $\vec{E}_{Z}(t)=-(1 / c)\left(d \vec{A}_{Z}(t) / d t\right)$. The vector potential $\vec{A}_{Z}(t)$ satisfies the following Maxwell equation:

$$
\frac{1}{c^{2}} \frac{\partial^{2}}{\partial t^{2}} \vec{A}_{Z}(t)-\frac{\partial^{2}}{\partial Z^{2}} \vec{A}_{Z}(t)=\frac{4 \pi}{c} \vec{J}_{Z}(t)
$$

where $\vec{J}_{Z}(t)$ is the electric current density at $Z$. The current is obtained from microscopic calculation of electron dynamics as described below.

We next turn to the microscopic calculation of electron dynamics at each macroscopic point, $Z$. We use the TDKS equation for it. A symbol $\vec{r}$ is used to denote the microscopic coordinates of electrons at macroscopic position $Z$. Since the wavelength of the laser pulse is much longer than the spatial scale of the electron dynamics in solids, we assume that the macroscopic electric field can be regarded as a spatially uniform field in the microscopic scale. We thus solve the following TDKS equation for electrons:

$$
i \frac{\partial}{\partial t} u_{b \vec{k}, Z}(\vec{r}, t)=\left[\frac{1}{2 m}\left(\vec{p}+\hbar \vec{k}+\frac{e}{c} \vec{A}_{Z}(t)\right)^{2}+V\right] u_{b \vec{k}, Z}(\vec{r}, t)
$$

where $u_{b \vec{k}, Z}(\vec{r}, t)$ is the time-dependent Bloch orbital that has the same periodicity as that of the crystalline solid, $u_{b \vec{k}, Z}(\vec{r}, t)=u_{b \vec{k}, Z}(\vec{r}+\vec{a}, t)$. We employ a standard Kohn-Sham Hamiltonian in the adiabatic local-density approximation in Eq. (2). A periodic potential $V$ includes 
electron-ion and electron-electron interactions. The electron-ion interaction is treated using a norm-conserving pseudo-potential [7] with a separable approximation [8]. The electronelectron interaction includes the Hartree potential and the exchange-correlation potential [9].

The macroscopic electric current, $\vec{J}_{Z}(t)$, is an essential quantity that connects two spatial scales together with the vector potential $\vec{A}_{Z}(t)$. The current is given by

$$
\vec{J}_{Z}(t)=\frac{1}{\Omega} \sum_{b \vec{k}} \int_{\Omega} d \vec{r} u_{b \vec{k}, Z}^{*}(\vec{r}) \vec{j}(t) u_{b \vec{k}, Z}(\vec{r}),
$$

where $\vec{j}(t)$ is the current operator and $\Omega$ is the volume of the unit cell.

Equations (1) and (2) are the basic equations of the multi-scale simulation. They are coupled: the vector potential $\vec{A}_{Z}(t)$ is an input and the electric current $\vec{J}_{Z}(t)$ is an output of Eq. (2), while the time propagation of $\vec{A}_{Z}(t)$ is described by Eq. (1) where $\vec{J}_{Z}(t)$ is brought from Eq. (2). As an initial condition, macroscopic electromagnetic fields are prepared in the vacuum region, while electron orbitals of all macroscopic grid points are prepared as the ground state. We note that, if we employ a perturbation theory assuming that the field described by $A_{Z}(t)$ is weak, the two equations are decoupled, resulting in the conventional macroscopic electromagnetism. Solving Eqs. (1) and (2) simultaneously without any perturbative expansion, we may describe propagation of macroscopic electromagnetic fields and microscopic electron dynamics for intense and ultrasort laser pulses. We call it the Maxwell +TDDFT multi-scale simulation.

\subsection{Illustrative example}

As an example of our simulation, we show a time evolution of an intense laser pulse irradiating normally on a diamond thin film of $800 \mathrm{~nm}$ thickness. Figure 2 shows snapshots of the electric field of the laser pulse. In the figure, the diamond thin film is shown by a gray area in $0<Z<0.8 \mu \mathrm{m}$. Other areas of $Z<0$ and $Z>0.8 \mu \mathrm{m}$ are vacuum. The upper panel shows the electric field when a part of the laser pulse reaches the film, while the lower panel shows the electric field when the laser pulse splits into transmitted (right to the film) and reflected (left to the film) waves. Since the reflection takes place at both front and rear surfaces of the thin film, there appear two reflected pulses, one reflected from the front and the other reflected from the rear surfaces. Red solid curve shows the electric field of the intense laser pulse whose maximum intensity in the incident pulse is $1.0 \times 10^{14} \mathrm{~W} / \mathrm{cm}^{2}$. Blue-dotted curve shows that of weak laser pulse whose maximum intensity is $1.0 \times 10^{10}$ $\mathrm{W} / \mathrm{cm}^{2}$. The pulse width is about $5 \mathrm{fs}$ in the FWHM. The mean frequency is $1.55 \mathrm{eV} / \hbar$ that is lower than the optical band gap of diamond. In our calculation using the adiabatic local density approximation for the exchange-correlation functional, the optical band gap is 5.6 $\mathrm{eV}$. The electric field of the weak pulse is multiplied by a factor of 100 so that the incident electric fields coincide.

As mentioned above, our simulation scheme results in the conventional macroscopic electromagnetism for weak fields, the blue-dotted curve corresponding to that limit. Differences between the red-solid and blue-dotted curves manifest effects of nonlinear electron dynamics induced by the intense laser pulse. 

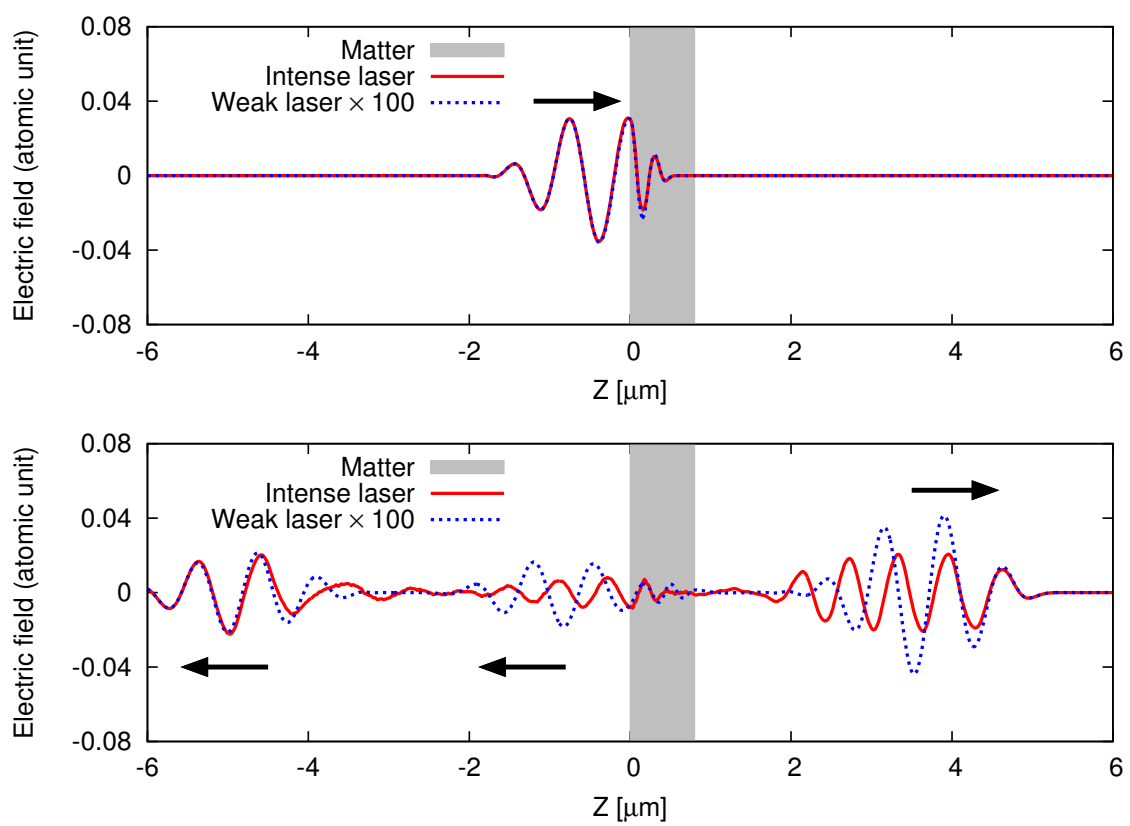

Figure 2: Snapshots of the electric-field propagation. The upper panel shows the electric fields when the fields start to irradiate on the diamond thin-film, while the lower panel shows those when the fields split into reflected and transmitted pulses. Red-solid line corresponds to the incident laser pulse of the intensity $10^{14} \mathrm{~W} / \mathrm{cm}^{2}$ and wave length of $800 \mathrm{~nm}$. Blue dotted line corresponds to a weak incident pulse of the intensity $10^{10} \mathrm{~W} / \mathrm{cm}^{2}$ for which the linear response approximation applies. The latter is multiplied by a factor of 100 so that the incident electric fields coincide with each other.

Looking at front parts of both transmitted and reflected waves, electric fields of intense and weak laser pulses almost coincide to each other. This is because the laser field at the front of the wave is always weak and does not induce any nonlinear phenomena. As the strength of the electric field increases, there appears large difference between intense and weak laser pulses due to nonlinear electron dynamics.

Looking at Fig. 2 in more detail, one may see that first one cycle of the transmitted electric field of intense pulse is just suppressed in intensity, without any phase shift. On the other hand, there is a substantial phase shift for second and later cycles of the transmitted wave. This indicates there are two kinds of nonlinear interactions. When the front part of the laser pulse reaches the surface of the film, electrons in the film are in their ground state. In this case, the nonlinear interaction works only to suppress the electric field of the laser pulse without any phase shift. On the other hand, once the strong electric field of the laser pulse excites electrons into conduction bands, the dielectric function of the solid is strongly modified. This causes the large change in the index of refraction and induces the phase shift. Comparing two reflected electric fields, one around $Z \simeq-5 \mu \mathrm{m}$ reflected at the front surface of the film and the other around $Z \simeq-1 \mu \mathrm{m}$ reflected at the rear surface, the former shows little phase shift while the latter shows much. This difference can also be explained by the 
change of the index of refraction.

\section{Numerical implementation, parallelization and computational performance}

We develop our own code named ARTED = Ab-initio Real-Time Electron Dynamics simulation code, for the Maxwell + TDDFT multi-scale simulation. In this section, we first describe numerical implementation of our code. We then describe the parallelization and the computational performance of the code.

\subsection{Propagation of macroscopic electromagnetic fields}

To start with, we describe numerical method to solve Eq. (1) for the macroscopic electromagnetic fields. Introducing uniform spatial grid for the macroscopic coordinate $Z$, we solve Eq. (1) using a finite difference method:

$$
\begin{aligned}
\vec{A}_{Z}(t+\Delta t)= & 2 \vec{A}_{Z}(t)-\vec{A}_{Z}(t-\Delta t) \\
& +c^{2} \Delta t^{2} \frac{\vec{A}_{Z+\Delta Z}(t)-2 \vec{A}_{Z}(t)+\vec{A}_{Z-\Delta Z}(t)}{\Delta Z^{2}}+4 \pi c \Delta t^{2} \vec{J}_{Z}(t),
\end{aligned}
$$

where $\Delta t$ is the time step and $\Delta Z$ is the grid spacing. We use a simple three-points formula that gives sufficient accuracy. To calculate a vector potential of next time step, $\vec{A}_{Z}(t+\Delta t)$, from that of $t$, we need the electric current at time $t, \vec{J}_{Z}(t)$. As will be described below, the electric current is provided from the microscopic calculation of the electron dynamics. The computational cost of this macroscopic electromagnetic fields is negligible once the electric current is provided.

\subsection{Time-evolution of microscopic electron orbitals}

We next turn to the time-evolution calculation of electron orbitals. At each macroscopic grid point $Z$, we prepare Bloch orbitals for electrons, $u_{b \vec{k}, Z}(\vec{r}, t)$. The orbitals have two indices. One is the band index $b$ and the other is the crystalline momentum $\vec{k}$ that is discretized in the first Brillouin zone. We employ three-dimensional Cartesian grids for microscopic coordinate $\vec{r}$. The grid spacing $h$ of the microscopic coordinate $\vec{r}$ is much smaller than $\Delta Z$. A nine points finite difference formula is used for both first and second spatial derivatives.

To evolve the orbitals according to the TDKS equation (2), we employ the Taylor expansion method $[5,10]$.

$$
u_{b \vec{k}, Z}(\vec{r}, t+\Delta t) \sim \sum_{n}^{N} \frac{\left(-i h_{Z}(t) \Delta t / \hbar\right)^{n}}{n !} u_{b \vec{k}, Z}(\vec{r}, t),
$$

where $h_{Z}(t)$ is the Kohn-Sham Hamiltonian at macroscopic point $Z$ :

$$
h_{Z}(t)=\frac{1}{2 m}\left(\vec{p}+\hbar \vec{k}+\frac{e}{c} \vec{A}_{Z}(t)\right)^{2}+V .
$$




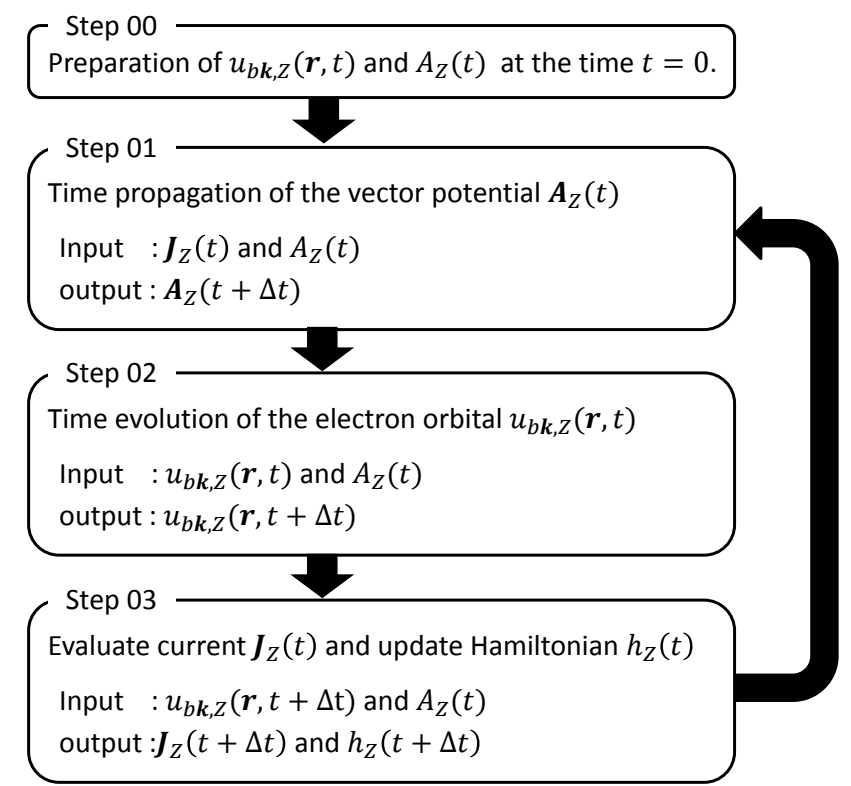

Figure 3: Flowchart of the multi-scale simulation.

In practice, we use a fourth-order expansion, $N=4$.

The Hamiltonian $h_{Z}(t)$ depends on time through two quantities. One is the electron density $\rho_{Z}(\vec{r}, t)$ which is calculated from electron orbitals $u_{b \vec{k}, Z}(\vec{r}, t)$ at the same macroscopic grid point $Z$. The other is the macroscopic vector potential $\vec{A}_{Z}(t)$ which is obtained by solving Eq. (4). We note that the update of the Hamiltonian does not require any direct communications among electron orbitals belonging to different macroscopic grid points. The data of different macroscopic grid points are exchanged only through the macroscopic vector potential $A_{Z}(t)$.

\subsection{Coupled dynamics of macroscopic and microscopic scales}

We next describe how we combine two procedure, Eq. (1) for macroscopic vector potential $\vec{A}_{Z}(t)$ and Eq. (2) for microscopic electron orbitals $u_{b \vec{k}}(\vec{r}, t)$. Figure 3 shows a brief summary of the procedures.

In the initial step 00, we prepare initial conditions. We solve the static Kohn-Sham equation in a unit cell of a solid and obtain the electron orbitals of the ground state. This part of the calculation is nothing but the ordinary band structure calculation with the density functional theory. The calculated electron orbitals are set as initial orbitals at every macroscopic grid point. The computational cost of this step is negligible, since it is much smaller than that of the time evolution part. As for the vector potential $\vec{A}_{Z}(t)$, we set an incident laser pulse in the vacuum region. We also calculate the density, current, and the Hamiltonian from the initial orbitals.

From the initial condition described above, we successively evolve $\vec{A}_{Z}(t)$ and $u_{b \vec{k}}(\vec{r}, t)$ in 
Macroscopic grids

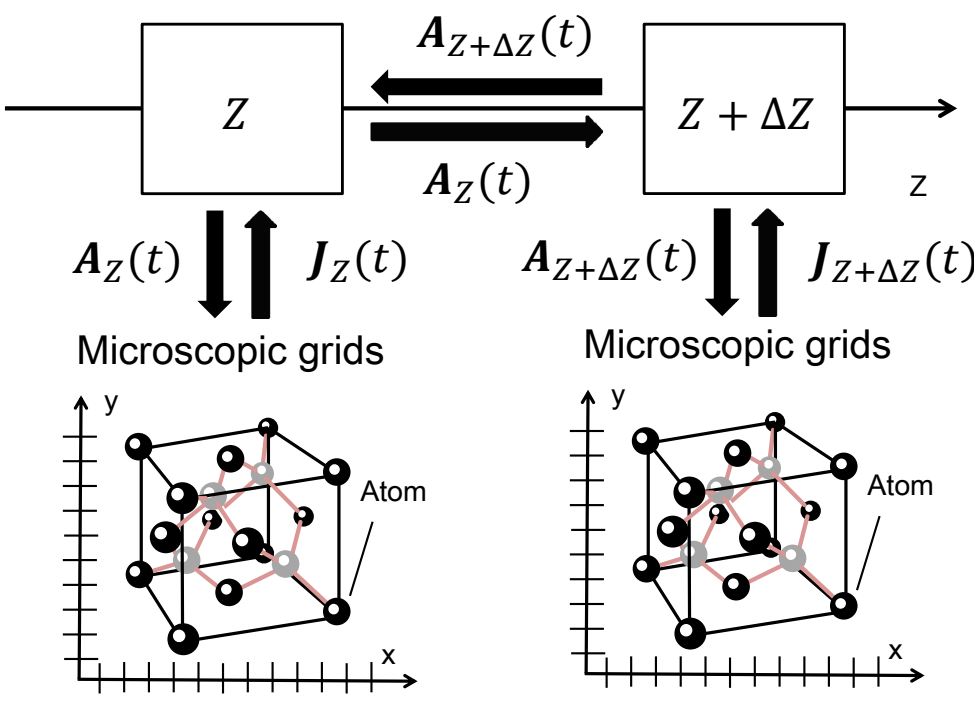

Figure 4: A schematic picture showing connections among grid systems. At each macroscopic gird points, the vector potential, $\vec{A}_{Z}(t)$, and electric current, $\vec{J}_{Z}(t)$, are exchanged between macroscopic and microscopic scales. The macroscopic grid points are connected to next neighbours by exchanging vector potentials, $\vec{A}_{Z \pm \Delta Z}(t)$.

turn. In step 01 , the vector potential $\vec{A}_{Z}(t)$ is evolved for a small time step $\Delta t$ using Eq. (4). To calculate $\vec{A}_{Z}(t+\Delta t)$ in this step, the electric current $\vec{J}_{Z}(t)$ is required as well as the vector potential of previous time.

In step 02 , electron orbitals $u_{b \vec{k}}(\vec{r}, t)$ are evolved for the time step $\Delta t$. We use the vector potential at $t+\Delta t / 2$ to calculate the orbitals at $t+\Delta t$ from those of $t$. The vector potential at $t+\Delta t / 2$ is approximated by $\left(A_{Z}(t+\Delta t)+A_{Z}(t)\right) / 2$. We note that $A_{Z}(t+\Delta t)$ is already calculated at step 01 . The time evolution of electron orbitals are achieved using the Taylor expansion method, Eq. (5). This step is the most time consuming procedure in the whole calculation, because the operation of the Hamiltonian $h_{Z}(t)$ on electron orbitals is computationally demanding. Fortunately, the time-evolution of the electron orbitals can be achieved independently for each orbital. Therefore we can efficiently parallelize the most time-consuming part.

In step 03, we calculate the density and the current at time $t+\Delta t$ from the electron orbitals $u_{b \vec{k}}(\vec{r}, t+\Delta t)$. The current will be used to evolve the vector potential $A_{Z}(t)$ in the next step. Using the density, we also construct the Hamiltonian $h_{Z}(t+\Delta t)$ that will be used to update the electron orbital in the next step 01 .

By repeating the steps 01-03, one can solve the coupled equations of the macroscopic Maxwell equation (1) and the microscopic TDKS equation (2) simultaneously. 


\subsection{Parallelization}

The present multi-scale simulation can be very efficiently computed in massively parallel machines. To understand it and to illustrate our parallelization strategy in practice, we first illustrate computational aspects of our simulation, stressing exchange of data required to proceed the calculation. Figure 4 shows data flows among macroscopic grid points. Between macroscopic grid points $Z$ and $Z+\Delta Z$, exchange of the vector potential data is required to evolve it by Eq. (4). There are mutual data flows between macroscopic and microscopic levels: the vector potential $\vec{A}_{Z}(t)$ is an input to solve the TDKS equation (2) in the microscopic level. The macroscopic current $J_{Z}(t)$ is calculated in the microscopic level and transferred to the macroscopic level. This is an input to solve the Maxwell equation (4). We note that there is no direct exchange of data between electron orbitals belonging to different macroscopic grid points.

In the practical implementation using MPI, we assign a communicator to each macroscopic grid point. The number of the communicators is equal to the number of macroscopic grid points. In principle, each communicator only need to have data of vector potential $A_{Z}(t)$ and current $J_{Z}(t)$ of the corresponding macroscopic grid point. In evolving the vector potential using Eq. (4), we need data of vector potential of nearest neighbour macroscopic grid points that requires point-to-point communication between communicators. In the present implementation of the ARTED, however, data of vector potential and current of all macroscopic grid points are shared among all the communicators. Then the macroscopic Maxwell equation (4) is redundantly solved in all the communicators. This redundancy does not harm computational performance because the one-dimensional Maxwell solver part is negligible in the total computational cost.

In addition to the macroscopic grid points, calculations of microscopic level, the time evolution of the TDKS equation, can also be very efficiently parallelized. There are two indices of the electron orbitals $u_{b \vec{k}, Z}(\vec{r}, t)$ that are also suited for parallelization: One is the band index $b$ and the other is the $k$-point. Compared with the parallelization in $Z$, there arise communications if we parallelize over indices $b$ and $\vec{k}$. At each time step, we need to sum up electron orbitals over $b$ and $\vec{k}$ to calculate density and current. The density is used to construct the time-dependent Hamiltonian and the current is the basic output of the microscopic calculation. These communications can be achieved as collective communications inside the communicator of each macroscopic grid point.

Figure 5 shows a schematic picture for the parallelization of the multi-scale simulation in the ARTED. In our present implementation, we can parallelize with MPI for macroscopic grid points $Z$ and $k$-points $\vec{k}$. We can also parallelize in hybrid way using both MPI and OpenMP: $Z$ and $\vec{k}$ are parallelized with MPI, while $\vec{k}$ and band index $b$ are parallelized with OpenMP.

In the simulation of laser pulse irradiation on diamond thin-film shown in Sec. II, there are 80 macroscopic grid points $Z$ in the film. At each macroscopic grid point, we employ $160 k$-points and 16 bands. We calculate it with hybrid parallelization, 6400 MPI processes and 8 OpenMP threads on each MPI process. The 80 macroscopic points and $160 k$-points are parallelized by MPI so that each MPI process takes care of two $k$-points. Since there are 16 orbitals in each $k$-point and we employ 8 OpenMP parallelization, each thread takes 


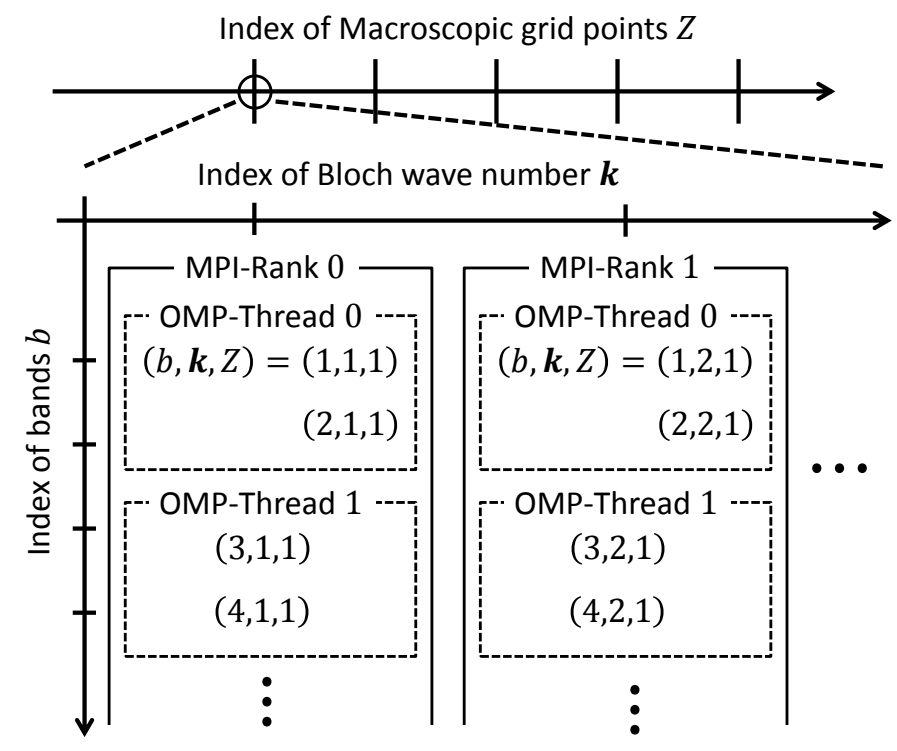

Figure 5: A Schematic picture of the parallelization of the multi-scale simulation in the ARTED. There are three quantities $(b \vec{k}, Z)$ that are indices of electron orbitals $u_{b \vec{k}, Z}(\vec{r}, t)$ and are subject to be parallelized.

care of 4 orbitals. In a cubic unit cell that contains 8 Carbon atoms, the three-dimensional Cartesian grids of $18 \times 18 \times 22$ points are employed in the microscopic scale. We do not employ any parallelization as to the microscopic spatial grids.

\subsection{Computational performance}

Figure 6 shows a strong-scaling parallel efficiency of the ARTED at K-computer. The data is taken for an irradiation of a laser pulse on bulk silicon. The number of macroscopic grid points is 160 , the electron orbitals at each macroscopic grid point has $1152 k$-points and 16 bands. When using 92160 cores, calculation is conducted by hybrid parallelization using 11520 MPI process times 8 OpenMP threads. Each thread takes care of 32 orbitals. As seen from Fig. 6, the ARTED shows a good parallel efficiently, more than $90 \%$ between 7680 and 92160 cores. This good efficiency reflects the fact that communication costs of our code is rather small even for a job using about 100,000 cores.

Let us remind of communications involved in the multi-scale calculation. As mentioned previously, we calculate propagation of the macroscopic vector potential $\vec{A}_{Z}(t)$ redundantly at every MPI processes. This requires an all-to-all communication of the current $\vec{J}_{Z}(t)$ at each time step. Since this is a rather small array of typically dozens of $k B$, it does not cost much. Inside each communicator, there is a all-to-all communication to calculate density and current from electron orbitals. The size of this communication depends on the number of bands $b, k$-points, and the number of microscopic grid points for coordinate $\vec{r}$. However, it is independent of the number of macroscopic grid points for $Z$. Therefore, once a material 


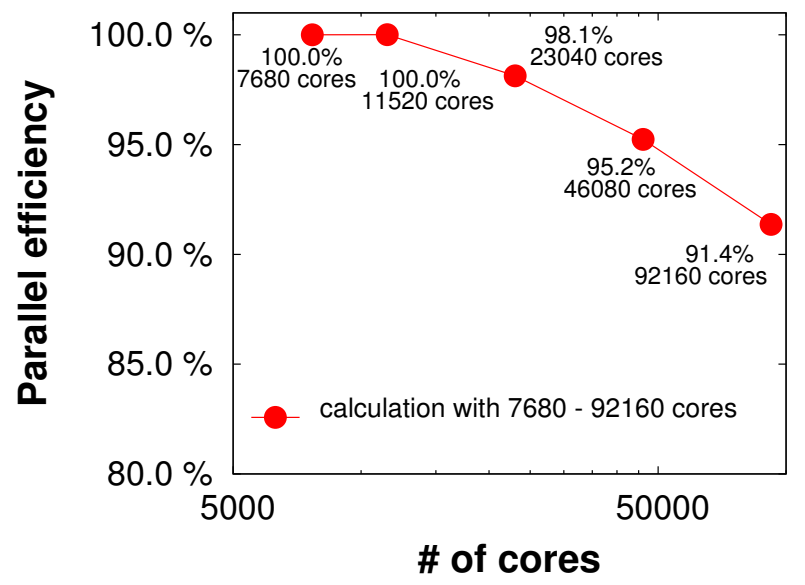

Figure 6: The strong-scaling parallel efficiency of the ARTED at K-computer.

to be calculated is set, the communication cost does not increase even if we increase the number of macroscopic grid points.

Figure 7 shows computing peromance of the ARTED. It is about $20 \%$ for cores from 7680 to 92160 . In our calculation, the most time-consuming part is the operation of the single-particle Hamiltonian, $h_{Z}(t)$, on electron orbitals, $u_{b \vec{k} Z}(\vec{r}, t)$ and it corresponds to an operation of a sparse Hermitian matrix on a complex vector. This operation can be achieved independently for all indices of $Z, \vec{k}$-points, and $b$. Therefore, the computational performance does not depend on the way to parallelize, as verified in the figure.

\section{Summary}

We have presented a new simulation method that is useful for interactions of intense, ultrashort laser pulses with solids. Since strong electromagnetic fields induce extremely nonlinear electron dynamics in solids, we need to solve a coupled dynamics of the macroscopic electromagnetic fields and the microscopic electron motion simultaneously. By virtue of large separation in length scale between macroscopic electromagnetic fields and microscopic electron dynamics, we can develop a multi-scale simulation in which the electron dynamics in microscopic scale is described using the time-dependent density functional theory.

As an application of the multi-scale simulation, we presented the interaction of intense laser pulse with a thin-film of diamond. We then describe in detail about the numerical implementation and the parallelization of the multi-scale method. Our multi-scale simulation has several variables that are quite adequate for parallel computations. They include the macroscopic grid points and orbital indices of electron dynamics calculation. In particular, the microscopic electron dynamics at each macroscopic grid pont can be calculated almost independently with very few communication costs. Because of these facts, good computational performance can be achieved. For example, the ARTED achieves more than $90 \%$ of 


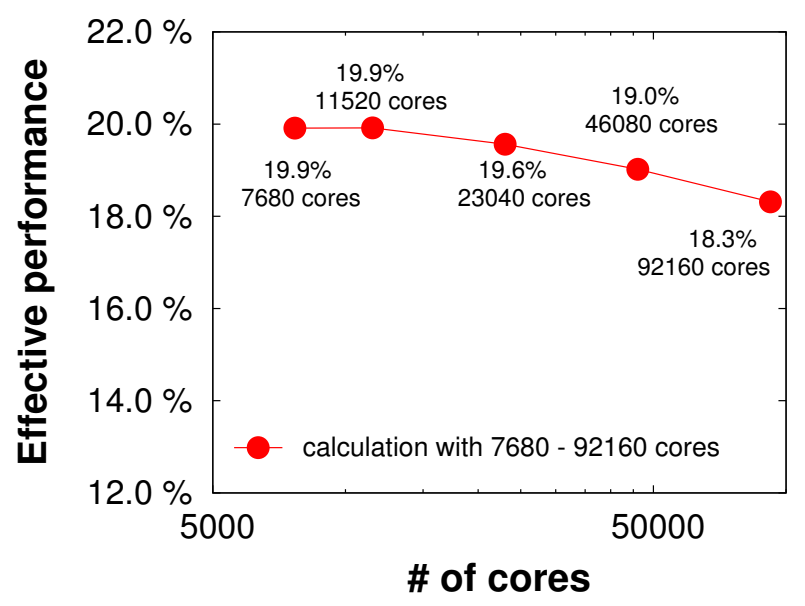

Figure 7: The computing performance of the ARTED at K-computer.

the parallel efficiency and about $20 \%$ of the computing efficiency, even if more than 90000 cores are employed in the calculation.

\section{Acknowledgement}

The numerical results are obtained by early access to the K computer at the RIKEN Advanced Institute for Computational Sciences. This research was supported by the Grant-inAid for Scientific Reseach Nos. 23340113 and 23104503, and the Grant-in-Aid for JSPS Fellows Grant No. 26-1511.

\section{References}

[1] E. Runge, E. K. U. Gross: Density-functional theory for time-dependent systems, Phys. Rev. Lett., 52 (1984), 997-1000.

[2] A. Schiffrin et al.: Optical-field-induced current in dielectrics, Nature, 493 (2013), 70-74.

[3] M. Schultze et al.: Controlling dielectrics with the electric field of light, Nature, 493 (2013), 75-78.

[4] K. Yabana et al.: Time-dependent density functional theory for strong electromagnetic fields in crystalline solids, Phys. Rev. B, 85 (2012), 045134.

[5] K. Yabana, G. F. Bertsch: Time-dependent local-density approximation in real time, Phys. Rev. B, 54 (1996), 4484-4487.

[6] G. F. Bertsch et al.: Real-space, real-time method for the dielectric function, Phys. Rev. $B, 62$ (2000), 7998-8002. 
[7] N. Troullier, J. L. Martins: Efficient pseudopotentials for plane-wave calculations, Phys. Rev. B, 43 (1991), 1993-2006.

[8] L. Kleinman, D. M. Bylander: Efficacious Form for Model Pseudopotentials, Phys. Rev. Lett., 48 (1982), 1425-1428.

[9] J. P. Perdew, A. Zunger: Self-interaction correction to density-functional approximations for many-electron systems, Phys. Rev. B, 23 (1981), 5048-5079.

[10] H. Flocard, S. E. Koonin, M. S. Weiss: Three-dimensional time-dependent HartreeFock calculations: Application to ${ }^{16} \mathrm{O}+{ }^{16} \mathrm{O}$ collisions, Phys. Rev. C, 17 (1978), 16821699. 